\title{
miR-9 and let-7g enhance the sensitivity to ionizing radiation by suppression of NFKB1
}

\author{
Himanshu Arora ${ }^{1 *}$, Rehana Qureshi ${ }^{1 *}$, \\ Shunzi Jin ${ }^{2 *}$, Ae-Kyoung Park ${ }^{1}$ \\ and Woong-Yang Park ${ }^{1,3}$ \\ ${ }^{1}$ Laboratory of Molecular and Genomic Medicine \\ Department of Biomedical Sciences \\ Seoul National University College of Medicine \\ Seoul 110-799, Korea \\ ${ }^{2}$ Ministry of Health Key Laboratory of Radiobiology \\ Jilin University \\ Changchun 130021, China \\ ${ }^{3}$ Corresponding author: Tel, 82-2-740-8241; \\ Fax, 82-2-744-4534; E-mail, wypark@ snu.ac.kr \\ *These authors contributed equally to this work. \\ DOI 10.3858/emm.2011.43.5.031
}

Accepted 31 March 2011

Available Online 5 April 2011

Abbreviations: IR, ionizing radiation; miRNA, microRNA

\begin{abstract}
The activation of nuclear factor-kappa B1 (NFKB1) in cancer cells may confer resistance to ionizing radiation (IR). To enhance the therapeutic efficiency of IR in lung cancer, we screened for microRNAs (miRNAs) that suppress NFKB1 and observed their effects on radiosensitivity in a human lung cancer cell line. From time series data of miRNA expression in $\gamma$-irradiated H1299 human lung cancer cells, we found that the expression of miR-9 was inversely correlated with that of NFKB1. Overexpression of miR-9 down-regulated the level of $\mathrm{NF} K \mathrm{BB} 1$ in $\mathrm{H} 1299$ cells, and the surviving fraction of $\gamma$-irradiated cells was decreased. Interestingly, let-7g also suppressed the expression of NFKB1, although there was no canonical target site for let-7g in the NFKB1 3' untranslated region. From these results, we conclude that the expression of miR-9 and let-7g could enhance the efficiency of radiotherapy for lung cancer treatment through the inhibition of NFKB1.
\end{abstract}

Keywords: ionizing radiation; let-7g; lung cancer; miR-9; NFKB1

\section{Introduction}

When living cells are exposed to ionizing radiation (IR), a series of alterations occurs including transformation, cell cycle distress, mutations, sisterchromatid exchanges, chromosome aberrations, DNA repair, and apoptosis (Preston, 2005; Amundson, 2008). The final outcomes of IR-exposed cells are determined by the cellular gene expression pattern (Amundson et al., 2003). Among the IR-responsive genes, the activation of nuclear factor-kappa B1 (NFкB1) following genotoxic stress allows DNA damage repair and cell survival (Janssens et al., 2005). The activation of NFКB1 in cancer cells may induce radioresistance, which frequently prevents successful treatment (Aggarwal et al., 2009).

Inhibition of $\mathrm{NF} \kappa \mathrm{B} 1$ increases sensitivity of cancer cells to the apoptotic action of chemotherapeutic agents and radiation exposure ( $\mathrm{Li}$ and Sethi, 2010). Various types of inhibitors targeting NFKB1 have been actively investigated as potential adjuvant therapeutics for lung cancer together with radiotherapy (Kunnumakkara et al., 2008). Radiosensitization induced by anti-inflammatory cytokines such as interleukin IL-4 and IL-10 in colorectal cancer was associated with NFкB1 inhibition (Voboril and Weberova-Voborilova, 2007). An endogenous inhibitor of gene expression, microRNA (miRNA), plays a significant role at the post-transcriptional gene regulation based on the 3' untranslated region (UTR) sequences. The alteration of miRNA expression upon IR may affect the gene regulation in the cellular response to radiation exposure (Chaudhry et al., 2010). It is important to find miRNAs targeting NF $\mathrm{NB}$ as a potential therapeutic approach to overcome radioresistance in cancer treatment.

Many studies have analyzed the transcriptional regulation of mRNAs and miRNAs in $\gamma$-irradiated cells to understand cellular responses to IR (Park et al., 2002; Weidhaas et al., 2007; Jeong et al., 2009). In this study, we screened the expression profiles of miRNA in $\gamma$-irradiated $\mathrm{H} 1299$ human lung cancer cell line to find miRNA targeting NFкB1. We found that miR-9 and let-7g could increase the sensitivity of $\mathrm{H} 1299$ cells to IR in vitro. Thus, we propose that the suppression of NF $\kappa B 1$ by miR-9 and let-7g may provide opportunities for both prevention and treatment of cancer. 
Table 1. List of microRNA profiles targeting NFKB1 in g-irradiated $\mathrm{H} 1299$ cells

\begin{tabular}{|c|c|c|c|c|c|c|c|}
\hline \multirow{2}{*}{ Genes } & \multicolumn{6}{|c|}{ Expression of genes after $\gamma$-irradiation Log2 [Signal intensity] } & \multirow{2}{*}{$\begin{array}{l}\text { Pearson's } \\
\text { correlation } \\
\text { coefficient }\end{array}$} \\
\hline & $\mathrm{Oh}$ & $2 \mathrm{~h}$ & $4 \mathrm{~h}$ & $8 \mathrm{~h}$ & $12 \mathrm{~h}$ & $24 \mathrm{~h}$ & \\
\hline NFאB1 & 8.95 & 9.01 & 9.31 & 9.43 & 9.36 & 9.00 & \\
\hline miR-424 & 6.32 & 6.00 & 6.29 & 4.23 & 4.98 & 5.44 & -0.65 \\
\hline miR-9 & 5.80 & 5.95 & 5.90 & 4.29 & 4.46 & 4.40 & -0.46 \\
\hline miR-195 & 7.84 & 7.38 & 8.32 & 6.87 & 7.09 & 7.20 & -0.24 \\
\hline miR-183 & 4.05 & 4.11 & 3.41 & 5.57 & 5.40 & 5.35 & 0.33 \\
\hline
\end{tabular}

\section{Results}

To identify miRNAs that suppress NFkB1 expression, we profiled miRNA in H1299 lung cancer cells at $0,2,4,8,12$, and 24 hours after $2 \mathrm{~Gy} \gamma$ irradiation. We filtered the list of miRNAs in a time series profile based on the target prediction using TargetScan (Lewis et al., 2005) and the correlation coefficient between NFKB1 and miRNAs. Among 328 human miRNAs in the microarray, we found that expression of miR-9, miR-424, and miR-195 was inversely correlated with expression of $\mathrm{NF} \kappa \mathrm{B} 1$ in $\gamma$-irradiated $\mathrm{H} 1299$ cells (Table 1). The expression patterns of these selected miRNAs are shown in a heatmap (Figure 1A). Among these selected miRNAs, we confirmed the expression of miR-9 and NFKB1 in $\gamma$-irradiated $\mathrm{H} 1299$ cells using real-time RT-PCR (Figures 1B and 1C). We used let- $7 \mathrm{~g}$ and miR-26b as controls, because these two miRNAs do not have binding sites in NFKB1 3'UTR. The expression of let-7g and miR-26b also decreased upon IR in H1299 cells, as did miR-9.

The miRNA miR-9 was previously found to be a candidate miRNA targeting NFKB1, (Bazzoni et al., 2009), but its effect on radiosensitivity was not examined. There is one defined miR-9 target site at position 29-35 of NFKB1 3' UTR, as shown in Figure $2 \mathrm{~A}$. We introduced a miR-9-expression vector into H1299 cells and then measured the expression of miR-9 using real-time RT-PCR. We used let-7g and miR-26b as controls to check the specific effect of miR-9 (Figure 2B). miR-9 inhibited $\mathrm{NF} \kappa \mathrm{B} 1$ expression in real-time RT-PCR as well as in western blot analysis (Figures $2 \mathrm{C}$ and 2D), indicating that a strong correlation between miR-9 and NFKB1 exists. To confirm that 3' UTR of $N F_{K B} 1$ has the binding site for miR-9 we cloned 3'UTR of the candidate gene downstream of the renilla luciferase reporter gene. MiR-9 target the $N F K B 1$ gene was confirmed by the decrement in the Renilla activity (Figure 2E). Interestingly, let-7g inhibited the expression of $N F \kappa B 1$ as much as miR-9, while miR-26b did not.

To study whether miR-9 could sensitize H1299 cells to IR, we first overexpressed miR-9 and
A

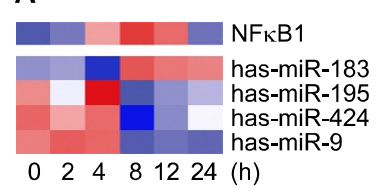

B
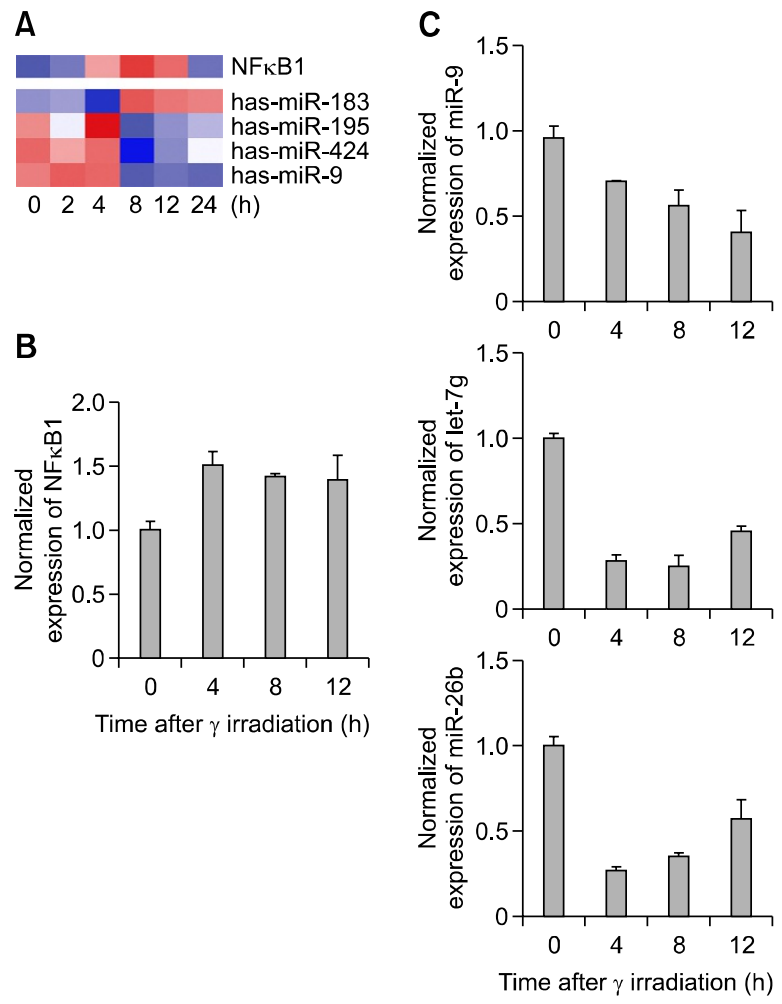

Figure 1. Expression patterns of $\mathrm{NF} \kappa \mathrm{B} 1$ and microRNAs in $\gamma$-irradiated H1299 cells. (A) Heatmap analysis shows the expression of NFKB1 and microRNAs targeting NF $\kappa B 1$ in $\mathrm{H} 1299$ cells upon ionizing radiation at 0 , 4,8 , and $12 \mathrm{~h}$. (B) The expression of NF B1 mRNA was quantitated with real-time RT-PCR at the indicated time. The values were normalized with GAPDH mRNA. (C) The expressions of miR-9, let-7g, and miR-26b were measured by real-time RT-PCR in $\gamma$-irradiated $\mathrm{H} 1299$ cells at the indicated time. U6B mRNA was used as a normalization control for microRNAs. All values are presented as mean $\pm S D$ from triplicate experiments.

measured cell survival upon $\gamma$-irradiation at $0,2,4$, and 6 Gy using the MTT assay (Figure $3 A$ ). We used let-7g and miR-26b as controls, since there are no binding sites for these miRNAs in NFKB1 3'UTR sequences. miR-9 suppressed the expression of NFKB1 at both the mRNA and protein level (Figure 2D), and significantly sensitized H1299 cells to IR in a survival analysis. Interestingly, let-7g 
A
3' aguaugucgaucuauUgguUUCu 5 ' has-miR-9
| | | | | | | | |||||

5' uccCACACCGUGUAAACCAAAGc 3' NFkB1

B

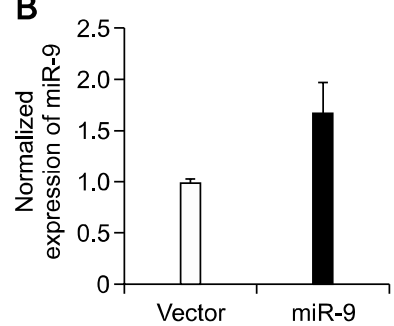

C

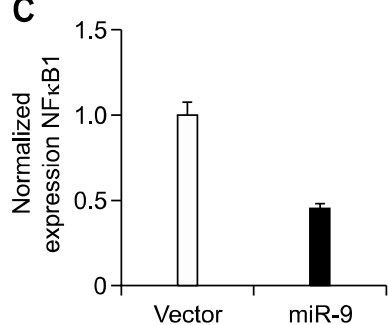

D

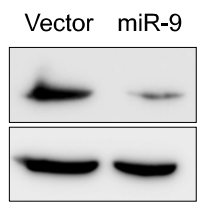

E

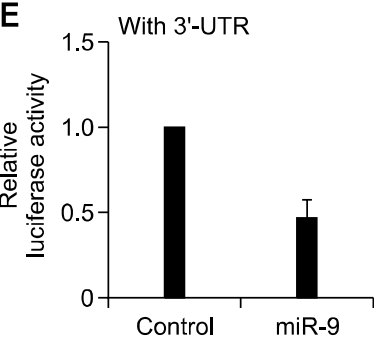

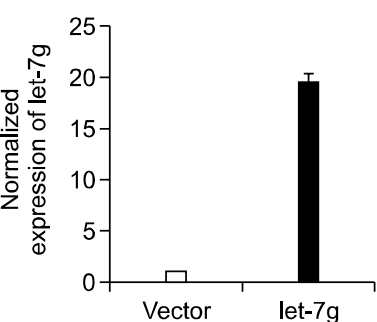
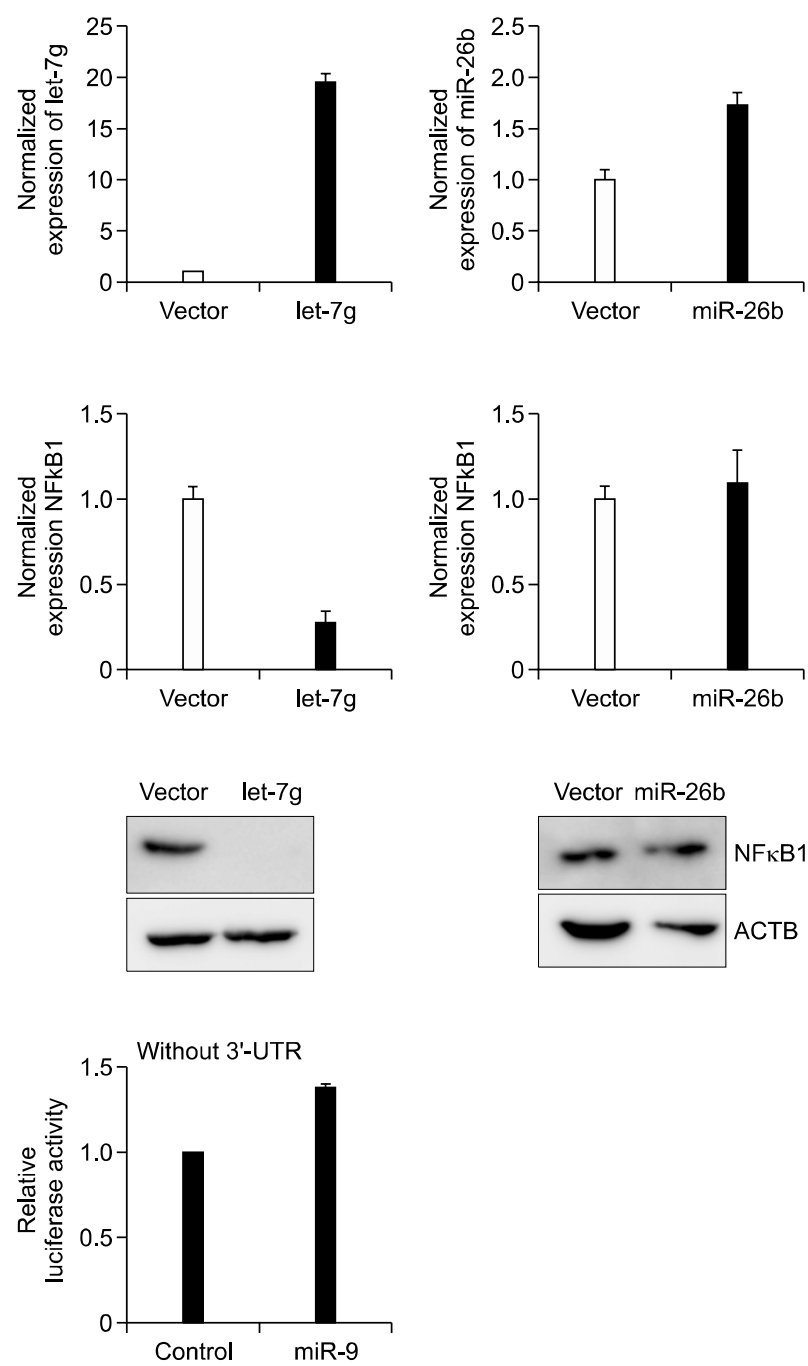

Figure 2. Suppression of NFKB1 by miR-9 and let-7g. (A) Target sequence of miR-9 in NF B1 3'UTR was predicted by TargetScan. (B) In miR-9, let-7g, and miR-26b transfected H1299 cells, the expression of each microRNA was confirmed by real-time RT-PCR. (C) The expression of NFKB1 mRNA in microRNA-transfected cells was measured by real-time RT-PCR. The relative NFKB1 expression levels were normalized against GAPDH and presented as mean \pm SD from triplicate experiments. (D) The protein levels of NFKB1 were also examined by western blot in microRNA-transfected cells. (E) Cells were transfected with the empty renilla luciferase reporter gene (psiCHECK2) or the reporter gene fused to the NFKB1 3' UTR. In addition, the cells were co-transfected with miR-9 or without miR-9. Results are expressed as relative light units (RLU) and were normalized with the luciferase activity expressed constitutively by the psiCHECK2 vector.

also suppressed the expression of $\mathrm{NF}_{\kappa \mathrm{B}} 1$ as much as miR-9 did, and also enhanced the sensitivity of $\mathrm{H} 1299$ cells to $\gamma$-irradiation. The expression of miR$26 \mathrm{~b}$ did not alter the expression of NFKB1 or cell survival. In order to testify the microRNA mediated sensitization in other cell lines we selected A549 cells and performed the MTT assay by first over- expressing miR-9 and then measuring the cell survival upon $\gamma$-irradiation at $0,2,4$, and 6 Gy respectively. It was found that has-miR-9 sensitized A549 cells thereby further supporting our hypothesis (Figure $3 \mathrm{C}$ ).

We also measured the expression patterns of NF $\kappa$ B1 in miR-9 and let-7g transfected cells upon 

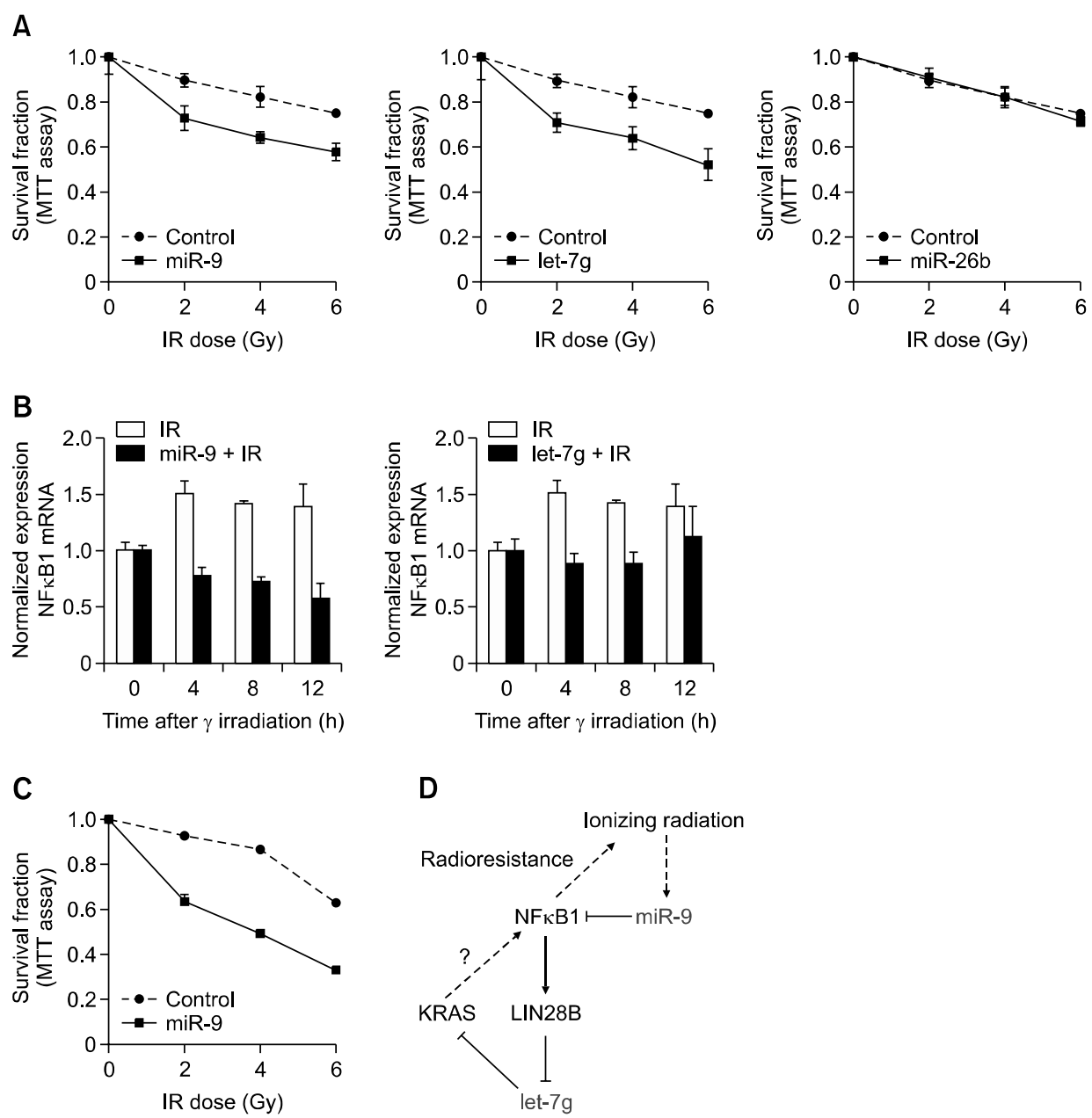

Figure 3. miR-9 and let-7g enhance the sensitivity to ionizing radiation in $\mathrm{H} 1299$ cells. (A) miR-9, let7g, and miR-26b were overexpressed and the cell survival fraction upon $\gamma$-irradiation was measured using MTT assay in H1299 cells. (B) The expression of NFKB1 was measured in microRNA-transfected cells after $\gamma$-irradiation. (C) miR-9 was overexpressed and the cell survival fraction upon $\gamma$-irradiation was measured using MTT assay in A549 cells. (D) miR-9 can directly control the expression of $\mathrm{NF \kappa B1}$, and the indirect regulation of $\mathrm{NF} \kappa \mathrm{B} 1$ by let-7g may be proposed to control radiosensitivity.

IR (Figure 3B). The increase in NF $\mathrm{NB} 1$ expression is inhibited in miR-9 and let-7g transfected cells, suggesting that the expression of NFผB1 in $\gamma$ irradiated cells was regulated by the down-regulation of miR-9 and let-7g. These results demonstrate that $\mathrm{miR}-9$ and let-7g suppress the expression of $N F \kappa B 1$ and enhance the sensitivity to IR in H1299 lung cancer cells.

\section{Discussion}

In $\gamma$-irradiated H1299 human lung cancer cells, expression of miR-9 was down regulated while the expression of $\mathrm{NF}_{\kappa \mathrm{B}} 1$ was up regulated. Both miR-9 and let-7g suppressed the expression of $N F \kappa B 1$, which resulted in increased sensitivity to
IR in the H1299 human lung cancer cell line. Given these results, we suggest that miR-9 and let-7g may play an important role in the response to ionizing radiation through the inhibition of $\mathrm{NF} \kappa \mathrm{B} 1$. IR can induce DNA damage by inducing progressive changes in cell survival, growth, and proliferation by affecting gene expression (Amundson et al., 2003). Previous reports suggest that radiation can change the expression pattern of some genes (Park et al., 2002; Smirnov et al., 2009). NFкB1 has been previously suggested to alter the sensitivity to radiation and chemotherapeutic agents (Janssens and Tschopp, 2006; Perkins and Gilmore, 2006). Here we show that NFKB1 is targeted by miR-9 and let-7g, which results in the increased sensitivity to IR. The let-7 family of miRNAs regulates expression of oncogenes, such as RAS, and is 
specifically down regulated in many cancer subtypes. Low levels of let-7 predict a poor outcome in lung cancer. The let-7 family of miRNAs is overrepresented in a class of miRNAs exhibiting altered expression in response to radiation. More strikingly, we can also create a radiosensitive state when the select let-7 family of miRNAs is overexpressed in lung cancer cells in vitro, whereas decreasing their levels causes radioresistance through control of the proto-oncogene homologue let-60/RAS and genes in the DNA damage response pathway (Weidhaas et al., 2007). The let-7 family of miRNAs can suppress resistance to anticancer cytotoxic therapy, including radiotherapy. In the present study, let-7g sensitized the H1299 cells but also suppressed the expression of NF $\kappa \mathrm{B} 1$. Recently, Iliopoulos et al. (2009) reported that let-7g can suppress the expression of RAS, and ultimately suppress the expression of NFКB1 (lliopoulos et al., 2009). In a previous report, we showed that increased let-7g suppressed KRAS in H1299 cells (Jeong et al., 2009). This study examined miRNAs in radioresistance lung cancer cell lines and showed that miR-9 and let-7g can sensitize the $\mathrm{H} 1299$ cells by interacting with $N F \kappa B 1$. Along with $\mathrm{NF} \kappa \mathrm{B} 1$ there are other member genes like Lin28B, KRAS etc. (not included in the present study) which might be playing a crucial role in the overall regulation by interacting with each other and with microRNAs (Figure 3D). It is likely that miRNAs act in a similar dominant manner in radiation-induced DNA damage responses in other cancer types as well.

\section{Methods}

\section{Cell culture and $\gamma$-irradiation}

H1299 human lung cancer cell lines were maintained in RPMI 1640 medium and A549 cell lines were cultured in Dulbecco's Modified Eagle's medium (DMEM, Sigma Aldrich, St Louis, MO) supplemented with $10 \%$ fetal bovine serum, $100 \mathrm{U} / \mathrm{ml}$ penicillin, $100 \mu \mathrm{g} / \mathrm{ml}$ streptomycin, and 2 $\mathrm{mM}$ L-glutamine. The cultured cells were either exposed to 0-10 Gy of radiation using a 4-MV linear accelerator (Clinac 4/100, Varian, Palo Alto, CA) or left irradiated as a negative control.

\section{MicroRNA microarray and statistical analysis}

miRNAs were extracted using the mirVanamiRNA isolation kit (Ambion, Austin, TX) according to the manufacturer's protocols. Purified miRNAs were labeled using the mirVanamiRNA Array Labeling Kit and coupled to the Cy5 Post-Labeling Reactive Dye (Amersham, GE Healthcare Bio-Sciences, Piscataway, NJ). The labeled samples were washed and hybridized in duplicate to mirVanamiRNABioarrays
(Ambion) using the mirVanamiRNABioarray Essentials Kit. Fluorescence intensities were processed and measured using the GeneChip scanner $30007 \mathrm{G}$ (Agilent Technologies, Santa Clara, CA). The levels of miRNA hybridization were determined using GenePix Pro 6.0 software as recommended by the manufacturer. The background-adjusted intensity for each miRNA was subjected to a global variance stabilization normalization (VSN) procedure (Huber et al., 2002). The miRNAs targeting NFKB1 were predicted using TargetScan, and Pearson's correlation coefficient was calculated between the expression levels of miRNA and NFKB1 transcript (Sung et al., 2010) .

\section{Constructs and transfection}

The precursor of the hsa-miR-9 was amplified from the H1299 genomic DNA using the primers 5'-CCGGAATTC CCTCAACTCCACTCGTGTCC-3' and 5'-ATTGCGGCCG CTGGGACTGTGACTCCTACCTG-3', and the resulting product was cloned into EcoR1/Not1 restricted pcDNA3 (Invitrogen, Carlsbad, CA). The precursor of the has-miR26b was amplified from the H1299 genomic DNA using the primers 5'CGGATGGGAATTGGATACAT3' and 5' AGCTAC CCTGACCACTGCTG 3'cloned into PGEMT easy vector (Promega, Madison, $\mathrm{WI}$ ). The resulting product was subcloned into EcoR1/Not1 restricted pcDNA3.

The expression vector for hsa-let-7g was provided $\mathrm{S}-\mathrm{H}$. Jeong (Jeong et al., 2009), and the construct was transfected using FuGENE HD (Roche Applied Science, Mannheim, Germany) according to the manufacturer's protocols. Total RNA for real-time PCR, protein for western blot, and cells for MTT assay were collected after $48 \mathrm{~h}$ of transfection.

\section{RNA preparation and quantitative real-time RT-PCR}

Total RNA was extracted from cell lines using the TRIzol method according to the manufacturer's protocol (Kwak et al., 2009). Total RNA was reverse transcribed to complementary DNA using Superscript II reverse transcriptase (Invitrogen) and oligonucleotide primers. Quantitative RTPCR for genes was performed in a reaction mixture containing complementary DNA, SYBR Premix Ex Taq (Takara Bio Inc., Shiga, Japan), and primers for each gene.

Quantitation of miRNAs was carried out using TaqMan microRNA assays (Applied Biosystems, Foster City, CA). The PCR amplification was conducted in reaction mixture using the TaqMan Universal PCR Master Mixture according to the protocol supplied by the manufacturer. Samples were analyzed with the ABI PRISM 7000 sequence detection system (Applied BioSystems). All PCRs were performed in triplicate, and the specificity of the reaction was determined by melting curve analysis at the dissociation stage. We synthesized specific primers for NFKB1 (forward 5'-CTGCTGACAATTTCCCACAC-3'; reverse 5'-GCTCTC TGAGC ACCTTTGGA-3'). The relative quantitative method was used for the quantitative analysis. The calibrator was the averaged $\triangle \mathrm{Ct}$ from the untreated cells and the endogenous controls were GAPDH for genes and U6B for miRNAs. 


\section{Western blotting}

Cells were harvested, and lysed, and proteins were separated on a SDS/polyacrylamide gel and transferred to a PVDF membrane (Bio-Rad, Hercules, CA). After blocking, the membranes were incubated with the primary antibodies anti-ACTB and anti-NFKB1 polyclonal antibody (Santa Cruz Biotechnology, Santa Cruz, CA). Membranes were extensively washed and incubated with a horseradish peroxidase-conjugated secondary antibody (Bio-Rad). The antigen-antibody complexes were visualized by West-QChemiluminescent Sub Kit Plus (BIOTANG, Waltham, MA).

\section{Luciferase assay}

The 3' UTRs of NFKB1 were fused to the renilla gene using the Xhol/Notl restriction sites of the psiCHECK2vector (Promega). A total of $8 \times 10^{4} \mathrm{H} 1299$ cells were cotransfected with $30 \mathrm{ng}$ of the indicated vector and $90 \mathrm{ng}$ of the pcDNA3 cloned miR-9 using Fugene(HD) Roche for 48 h. Luciferase assays were performed using the DualLuciferase assay (Promega). Normalization of the Renilla expression was performed using the luciferase gene present on the psiCHECK2 vector.

\section{MTT assay}

H1299 cells were transfected with miR-9, miR26b, and let7g expression vector and A549 cells were transfected with miR-9 expression vector. Forty-eight hours after transfection, cells were seeded at 5,000/well into 96-well plates and exposed to various doses of IR at 0, 2, 4, and $6 \mathrm{~Gy}$. After $\gamma$-irradiation, the cells were incubated for 5 days, MTT (3-(4, 5-dimethylthiazole)-2, 5-diphenyltetrazoliumbromide) assay reagents were added, and the absorbance was measured at $560 \mathrm{~nm}$. All experiments were repeated at least three times, and data are presented as mean \pm standard deviation (SD). The Student's $t$-test was used to compare the means of the different groups. A $P$-value of $<0.05$ was considered statistically significant.

\section{Acknowledgements}

The authors thank members of the Laboratory of Molecular and Genomic Medicine for discussions and H.-J. Jeon for help with miRNA arrays. This paper is supported by a grant from Korea Science and Engineering Foundation (KOSEF), the Ministry of Education, Science and Technology, Korea (M20706000020-07M0600-02010 to W.Y.P), BRL (Basic Research Laboratory) program through the National Research Foundation of Korea funded by the Ministry of Education, Science and Technology (2010-0001200) and by the Korea Healthcare technology R\&D Project, Ministry for Health, Welfare \& Family Affairs (A084022).

\section{References}

Aggarwal BB, Vijayalekshmi RV, Sung B. Targeting inflammatory pathways for prevention and therapy of cancer: short-term friend, long-term foe. Clin Cancer Res 2009;15:
425-30

Amundson SA. Functional genomics in radiation biology: a gateway to cellular systems-level studies. Radiat Environ Biophys 2008;47:25-31

Amundson SA, Bittner M, Fornace AJ Jr. Functional genomics as a window on radiation stress signaling. Oncogene 2003;22:5828-33

Bazzoni F, Rossato M, Fabbri M, Gaudiosi D, Mirolo M, Mori L, Tamassia N, Mantovani A, Cassatella MA, Locati M. Induction and regulatory function of miR-9 in human monocytes and neutrophils exposed to proinflammatory signals. Proc Natl Acad Sci USA 2009;106:5282-7

Chaudhry MA, Kreger B, Omaruddin RA. Transcriptional modulation of micro-RNA in human cells differing in radiation sensitivity. Int J Radiat Biol 2010;86:569-83

Huber W, von Heydebreck A, Sultmann H, Poustka A, Vingron M. Variance stabilization applied to microarray data calibration and to the quantification of differential expression. Bioinformatics 2002;18 Suppl 1:S96-104

Iliopoulos D, Hirsch HA, Struhl K (2009). An epigenetic switch involving NF-kappaB, Lin28, Let-7 MicroRNA, and IL6 links inflammation to cell transformation. Cell 2009;139:693-706.

Janssens S, Tinel A, Lippens S, Tschopp J. PIDD mediates NF-kappaB activation in response to DNA damage. Cell 2005;123:1079-92

Janssens S, Tschopp J. Signals from within: the DNAdamage-induced NF-kappaB response. Cell Death Differ 2006;13:773-84

Jeong SH, Wu HG, Park WY. LIN28B confers radioresistance through the posttranscriptional control of KRAS. Exp Mol Med 2009;41:912-8

Kunnumakkara AB, Diagaradjane P, Guha S, Deorukhkar A, Shentu S, Aggarwal BB, Krishnan S. Curcumin sensitizes human colorectal cancer xenografts in nude mice to gamma-radiation by targeting nuclear factor-kappaBregulated gene products. Clin Cancer Res 2008;14:2128-36

Kwak H, Jeong KC, Chae MJ, Kim SY, Park WY. Flavonoids inhibit the AU-rich element binding of HuC. BMB Rep 2009;42:41-6

Lewis BP, Burge CB, Bartel DP. Conserved seed pairing, often flanked by adenosines, indicates that thousands of human genes are microRNA targets. Cell 2005;120:15-20

Li F, Sethi G. Targeting transcription factor NF-kappaB to overcome chemoresistance and radioresistance in cancer therapy. Biochim Biophys Acta 2010;1805:167-80

Park WY, Hwang CI, Im CN, Kang MJ, Woo JH, Kim JH, Kim YS, KimH, Kim KA, Yu HJ, Lee SJ, Lee YS, Seo JS. Identification of radiation-specific responses from gene expression profile. Oncogene 2002;21:8521-8

Perkins ND, Gilmore TD. Good cop, bad cop: the different faces of NF-kappaB. Cell Death Differ 2006;13:759-72

Preston RJ. Radiation biology: concepts for radiation protection. Health Phys 2005;88:545-56

Smirnov DA, Morley M, Shin E, Spielman RS, Cheung VG. 
Genetic analysis of radiation-induced changes in human gene expression. Nature 2009;459:587-91

Sung HY, Wu HG, Ahn JH, Park WY. Dcr3 inhibit p53dependent apoptosis in gamma-irradiated lung cancer cells. Int J Radiat Biol 2010;86:780-90

Voboril R, Weberova-Voborilova J. Sensitization of colore- ctal cancer cells to irradiation by IL-4 and IL-10 is associated with inhibition of NF-kappaB. Neoplasma 2007;54:495-502

Weidhaas JB, Babar I, Nallur SM, Trang P, Roush S, Boehm M, Gillespie E, Slack FJ. MicroRNAs as potential agents to alter resistance to cytotoxic anticancer therapy. Cancer Res 2007;67:11111-6 